\title{
AMPLIAÇÃO DA ACESSIBILIDADE E SEGURANÇA DOMICILIAR PARA IDOSOS: UMA APLICAÇÃ̃O DO DESIGN DE SERVIÇO
}

Marcella Lomba Nicastro (marcellaln@gmail.com) - Programa de Pós-Graduação em Design, Universidade Federal do Paraná.

Luize Andreazza Bussi (luize@arqlb.com.br) - Programa de Pós-Graduação em Design, Universidade Federal do Paraná.

Cesar Augusto Mendes de Queiroz (cesarquierozprojetos@gmail.com) - Programa de Pós-Graduação em Design, Universidade Federal do Paraná.

Prof. Dr. Aguinaldo dos Santos (asantos@ufpr.br) - Programa de Pós-Graduação em Design, Universidade Federal do Paraná.

\section{RESUMO}

Com o envelhecimento da população tem se demandado cada vez mais a busca de soluções para garantir a qualidade de vida e a manutenção da capacidade funcional dos idosos. Soluções orientadas ao ambiente construído recebem atenção especial. Convencionalmente tem se desenvolvido tais soluções com ênfase predominantemente orientada aos artefatos, havendo carência de maior volume de investigações sobre a dimensão dos serviços no atendimento das necessidades do idoso no ambiente construído. Neste contexto, o presente artigo apresenta um conceito de serviço, desenvolvido a partir de uma abordagem de "design science", com foco na garantia de maior acessibilidade e segurança para o idoso no ambiente construído.

Palavras chave: Idoso; moradia; segurança; acessibilidade; design de serviço

Área: O design e o desenvolvimento de produtos e serviços

\section{INTRODUÇÃO}

Este artigo pretende trazer luz e expor através de dados, pesquisas e ferramentas de Design de Serviço, o contexto, a necessidade $\mathrm{e} a$ aplicação de um serviço voltado à moradia do Idoso.

Convencionalmente, tem sido desenvolvido soluções com ênfase predominantemente orientada aos artefatos, havendo carência de maiores investigações sobre a dimensão dos serviços no atendimento das necessidades do idoso no ambiente construído. Neste contexto, o presente artigo dirige atenção à melhoria de qualidade de vida, à manutenção da capacidade funcional e às condições de segurança e acessibilidade do idoso em sua moradia. Assim sendo, serão analisados de forma sistêmica, alguns dos fatores sob a abordagem do "design science".

\subsection{Definições e cenários}

Segundo a Organização Mundial de Saúde (OMS) para os países em desenvolvimento, considera-se "idoso" o indivíduo com 60 anos ou mais. A partir de 1963, a OMS subdivide a velhice em estágios, considerando "idoso" o indivíduo com idade entre 60 e 74 anos, "ancião" entre 75 e 90 anos e como "velhice extrema" o estágio das pessoas com mais de 90 anos (WEINECK, 1991). Considera-se neste artigo, o mesmo critério entendido pelo Instituto 
Brasileira de Geografia e Estatística (IBGE) que, por sua vez, segue as denominações estabelecidas pela OMS.

O crescimento da população de idosos, em números absolutos e relativos, é um fenômeno mundial e está ocorrendo a um nível sem precedentes. A população de idosos no Brasil, segundo relatório do IBGE em 2015 atingiu 14,30 \% representando um aumento de 9,8\% comparados a 2005.

Expectativa ou esperança de vida, corresponde à quantidade de anos, em média, que uma determinada população vive. Esse item, é um importante indicador social que serve para avaliar a qualidade de vida de uma população de um determinado lugar, seus aspectos regionais e sociais. Fatores sociais relacionados ao aumento da expectativa de vida, referemse diretamente às taxas de $\mathrm{s}$ taxa de fecundidade, natalidade e mortalidade.

A partir da análise deste prognóstico levantam-se algumas questões: Como viverão estes novos idosos? Quais são as necessidades para que tenham uma vida com real qualidade a começar por sua moradia?

\subsection{Idoso e sua moradia}

Dados do IBGE, do Portal do envelhecimento e da FAU (Faculdade de Arquitetura e Urbanismo da USP), confirmam que é cada vez maior o número de idosos que vivem sozinhos, que associam ao morar individualmente a proteção de sua autonomia e o direito de cuidar de suas próprias vidas neste período, no qual a perspectiva é muito mais remota e volta-se muito mais ao passado, que ao futuro de vida longínqua.

Buscando uma relação mais pessoal dos profissionais com os usuários Mello (2007) menciona diferentes tipos de serviços de atenção ao idoso, de acordo com a Portaria 73 do Ministério da Saúde (2001), que prioriza a permanência do idoso na família e considera a institucionalização como a última alternativa.

Orientados pela Norma Brasileira de Acessibilidade $n^{\circ}$ 9050/2015, utilizando-se de cartilhas de prevenção de acidentes a idosos, pesquisas secundárias sobre moradia, segurança domiciliar e aspectos cognitivos associados ao idoso, levanta-se a avaliação multidisciplinar e sistêmica, peculiares ao Design de Serviços, na criação de um serviço, voltando o olhar ao espaço da moradia com atenção similar à dedicada à qualidade de vida física e mental. Entende-se, neste contexto, que a interpretação do termo qualidade de vida refere-se à atenção especial à saúde que, segundo a OMS a define por: "um estado de completo bem-estar físico, mental e social e não consistindo somente da ausência de uma doença ou enfermidade. “

As quedas em pessoas com mais de 60 anos assumem diariamente dimensões quase epidêmicas no Brasil, de acordo com o Ministério da Saúde. Neste quadro, as mulheres representam a maioria dos idosos internados pois, segundo geriatras, ficam mais vulneráveis por causa da osteoporose, da menopausa que promove a perda de massa óssea. Além dos casos citados, identificam-se como causas frequentes de quedas, problemas de visão, dificuldades auditivas, perda de musculatura e uso de medicamentos.

Até mesmo no aspecto social, as quedas podem significar grandes prejuízos na qualidade de vida dos idosos pois em geral causam dependência de terceiros, reclusão e consequentemente 
quadros de isolamento e depressão, quando não leva a perdas fatais decorrentes de hemorragias, traumatismos e fraturas de fêmur, citada como a mais comum causa de mortes.

Reduzir ao máximo os fatores de predisposição, tanto os relacionados aos indivíduos, quanto ao ambiente construído, constituem uma atitude preventiva ideal. No entanto, quando ocorrem as quedas, além dos fatores físicos a serem tratados, será necessário prevenir um segundo e subsequente episódio e curar as consequências psicológicas resultantes da primeira queda. $\mathrm{O}$ medo de tornar a cair acaba limitando, de modo inconsciente, os movimentos sendo necessários cuidados para a reabilitação da coordenação motora, da flexibilidade muscular, da automatização dos movimentos, bem como, da autoconfiança.

Considerando o alto número de idosos que vivem sozinhos e que, grande maioria são acidentes domésticos, faz-se necessário a avaliação das condições que este "lar" oferece para o bem-estar e segurança do idoso e a garantia da manutenção de sua autonomia. Pesquisas indicam que, desde pequenas adaptações na moradia, tais como retirada de objetos do caminho diário entre os ambientes de maior permanência, adaptação de mobiliário à alturas ergonômicas, ajustes nos estofados, seja em altura ou densidade, colocação de barras de apoio e antiderrapantes nos tapetes, até as mais significativas como mudanças estruturais no imóvel por exemplo, o aumento de aberturas para passagem de cadeiras de rodas, criação de rampas para substituir escada entre outras, podem contribuir significativamente para a melhor permanência nas residências e tornando a moradia acessível e segura.

A partir desta e de outras pesquisas secundárias que se utilizam de técnicas para identificação de demandas dos maiores de 60 anos, reforçou-se a motivação em tratar com especial atenção idosos pertencentes ao primeiro ciclo desta fase. Considera-se que a atitude preventiva e ainda melhor se preditiva, pode contribuir positivamente na promoção de um envelhecimento com qualidade. Através do caráter de multidisciplinaridade do Design de Serviços, da análise holística de fatores visa-se a criação de um serviço diferenciado de especial e efetiva contribuição.

\section{DESIGN DE SERVIÇOS}

Segundo Mager (2007) nas últimas décadas a base da economia das indústrias nos países ocidentais vem mudando de produtos manufaturados para fornecimento de informações e serviços. Neste cenário a necessidade do Design de Serviços vem preencher a lacuna de atendimento das necessidades do usuário que somente a execução de artefatos não atendia. A autora explica que o Design de Serviço está ligado a funcionalidade e a forma dos serviços a partir da perspectiva do usuário, com o objetivo de "assegurar que as interfaces dos serviços sejam úteis, usáveis e desejáveis sob o ponto de vista dos clientes e eficazes e eficientes e distintos sob o ponto de vista do prestador". No Design de Serviço o usuário participa ativamente do processo de entrega do serviço como co-criador das soluções.

Segundo Moritz (2005) o Design de Serviço pode ser compreendido também como a experiência de um serviço, bem como, o projeto do processo e da estratégia para entregá-lo. Diz respeito a entender o cliente, a organização e o mercado, desenvolver ideias, traduzi-las em soluções possíveis de implementá-las considerando todo o ciclo de vida do serviço e oportunidade de melhorias contínuas. Considera-se que design de serviços é uma "nova abordagem multidisciplinar de expertise". O designer de serviços seria responsável por criar e 
moldar as interfaces de contato com o cliente e projetar todos os detalhes da jornada do cliente que é um dos conceitos chave no entendimento do Design de Serviço (BLOMKVIST, 2011).

O Design de Serviço visto como uma abordagem centrada no usuário, possui métodos especiais de pesquisa que permitem aos clientes contribuir com o projeto de design. Vislumbra uma visão sistêmica sobre o projeto de serviços integrando produtos, processos e pontos de contato (DAMÁZIO, 2010).

\subsection{Design de serviços para idosos}

O presente artigo é resultado do trabalho prático realizado durante a disciplina de mestrado Design de Serviço na UFPR. Caracterizado por uma abordagem qualitativa, trata-se de um estudo exploratório que pretende contribuir para a compreensão do Design de Serviço sobre o tema envelhecimento e a importância de uma abordagem de prevenção e cuidado com os idosos. Refere-se ao trabalho de criação de um Serviço Preventivo à Moradia de Idosos que se baseou nas definições, conceitos e fundamentos do Design de Serviço. Entende-se a relevância de se pensar soluções específicas, personalizadas e diferenciadas para este público e promover serviços adequados às reais necessidades deste usuário. Entende-se também que a multidisplinaridade característica do Design de Serviço é um fator importantíssimo na consideração e formulação de análises construtivas de todos os itens pertinentes ao idoso, à sua moradia e os cuidados preventivos em ambos os casos.

Esta visão vem de encontro com a conceituação de envelhecimento que é ampla e complexa, na medida em que este processo é multifacetado e apresenta diversas dimensões: cronológica, biológica, social, econômica e cultural (QUEIROZ; LEMOS, 2002). Ao se considerar o público idoso torna-se primordial a utilização de determinantes de qualidade tais como: atender com cortesia, educação, cuidado à detalhes práticos e funcionais dos procedimentos bem como atenção tanto aos idosos quanto aos seus pertences, neste estudo a sua moradia; construir relação de confiabilidade conquistando-a gradativamente para que possa haver a cooperação do usuário e assim o melhor resultado do serviço. Esta confiança gradativa, neste trabalho está sendo proposta como a identificação de necessidades a serem atendidas, a apresentação de diagnóstico de ações de melhorias e a educação preventiva sobre segurança e acessibilidade do serviço. Também se faz atender de forma personalizada, eficiente e de comunicação simples, porém refinada para a melhor recepção e aceitação das soluções propostas no serviço.

Percebe-se pelo estudo bibliográfico e aplicabilidade, que o serviço proposto destinar-se-ia ao resultado neste caso funcional através da interação e participação do usuário a sensação de bem-estar, segurança e acessibilidade estaria promovida relacionada ou não ao uso de artefatos de proteção ou apenas à pequenas adequações e sugestões técnicas para melhoria preventiva das condições da moradia atual.

\section{MÉTODO DE PESQUISA}

O método utilizado para realização deste trabalho está baseado na revisão sobre métodos e ferramentas de Design de Serviços, com destaque para Moritz (2005) que propõe uma lista de seis etapas de atividades: SD Understanding, SD Thinking, SD Generating, SD Filtering, SD 
Explaining and SD Realising. Segundo Moritz, esta é uma maneira prática para entender e explorar o Design de Serviços, mas ressalta que trata-se de um modelo genérico com as principais sugestões de métodos e ferramentas para realizar as atividades, que precisam ser avaliadas conforme cada projeto.

Figura 1. Método de pesquisa. Fonte: Baseado em MORITZ (2005)
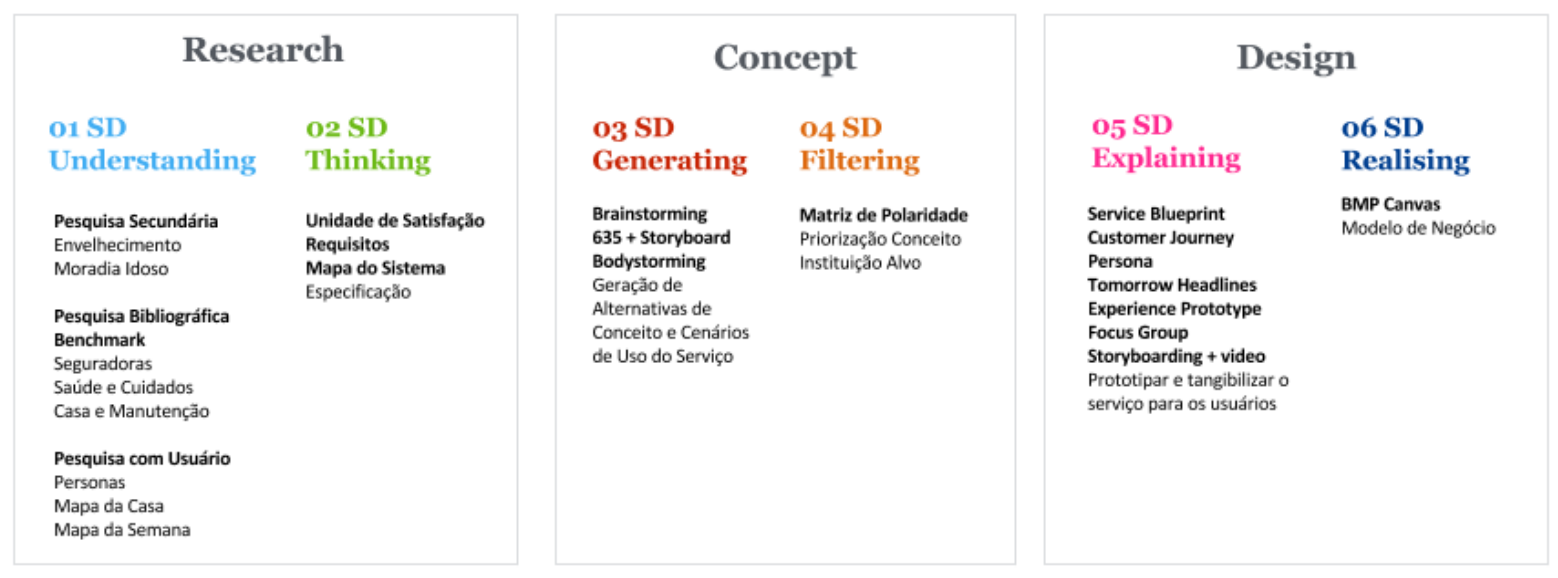

A etapa de pesquisa inicial (SD Understanding), buscou levantar informações sobre o contexto do tema abordado, explorando desafios e oportunidades para o projeto através das atividades Pesquisa Secundária e Benchmark. A revisão bibliográfica, fundamentada no conceito de acessibilidade e envelhecimento, apresentou as limitações comumente impostas pelo avanço da idade e seu impacto na manipulação do ambiente em que o idoso está inserido, especialmente o domicílio.

Com as informações coletadas nas pesquisas anteriores, foi possível representar os atributos dos potenciais usuários para o novo serviço através da ferramenta Persona. A reflexão inicial ajudou a identificar a necessidade de uma abordagem menos formal e mais lúdica para a interação com os usuários e facilitar sua participação no processo de design. Desta forma, a partir da ferramenta Storytelling (SERVICE DESIGN TOOLS, 2009), conhecida por dar suporte ao exploração da idéia do serviço através do uso de palavras onde o narrador ilustra a história da solução; duas ferramentas foram adaptadas: Mapa da Casa e Mapa da Semana, com o objetivo de mapear a rotina do idoso em sua própria residência. Utilizando um mapa que representa a planta da residência do usuário idoso e um calendário que representa os dias da semana, o usuário é orientado a contar histórias sobre seu dia dia, utilização dos cômodos, dificuldades que já teve e como lidou com elas. Trata-se de uma ferramenta simples, mas que ajuda a guiar a conversa com os usuários e deixá-los mais a vontade para falar sobre a relação com a moradia.

Para complementar a pesquisa com usuário, também foram realizadas Entrevistas com Especialistas em Gerontologia e visitas de Observação a Lares de Idosos na cidade de Curitiba.

A etapa de análise e direcionamento estratégico do projeto (SD Thinking) foi conduzida a partir da compreensão dos usuários e do mercado, identificando oportunidades em serviços 
voltados para moradia de idosos. Deste modo, foram apontados Requisitos, Unidade de Satisfação e Mapa do Sistema para o design do serviço.

Para a geração de alternativas e desenvolvimento do conceito para o novo serviço (SD Generating, SD Filtering), foram realizadas sessões de co-criação e seleção das ideias, considerando as necessidades orientadas a diferentes aspectos de vida dos idosos como segurança e acessibilidade, conforto, lazer e social, cognitivo e serviços. Destaca-se o uso da técnica criativa chamada Método 635 (ROHRBACH, 1953), oriunda da organização da equipe em grupo de 6 pessoas que geram 3 idéias em 5 minutos. A aplicação desta técnica ajudou a equipe desenhar propostas de solução como também a identificar características recorrentes que a solução deveria adotar, tais como moradia segura, assistência pessoal e espaço conceito.

A partir das idéias geradas com o Método 635, foi utilizado o método Bodystorming (SCHLEICHER; JONES; KACHUR, 2010), onde a equipe pode vivenciar as idéias geradas interagindo fisicamente com cenários ou objetivos reais. A experimentação das soluções para o novo serviço através deste método, favorece a empatia e o aprendizado sobre como melhor abordar o design sob a perspectiva de quem está vivenciando uma situação.

O novo conceito de serviço foi especificado e validado com os usuários na etapa de design (SD Explaining). As atividades realizadas nesta etapa visam tangibilizar as idéias e conceitos, mapeando os processos e ilustrando os cenários de uso e pontos de contato do serviço. Notase que a especificação da nova proposta de serviço ocorre de forma incremental e iterativa ao longo das atividades, com destaque para o Service Blueprint (BITNER; OSTROM; MORGAN, 2008) que constitui um ponto de referência comum para todas as partes interessadas em alcançar um modelo de serviço bem resolvido. O Service Blueprint relaciona de forma sequencial as operações do serviço, demonstrando as ações dos usuários e pontos de contato, com atividades e processos do prestador do serviço. Trata-se de uma ferramenta fundamental para entendimento da lógica do serviço.

Outro ponto importante nesta etapa é a participação do usuário no processo de prototipação, contribuindo para o resultado final da experiência do serviço.

Apesar deste estudo possuir restrições de escopo acadêmico e não explorar em profundidade outros aspectos estratégicos e de negócios do serviço, para a última etapa do trabalho foi desenvolvida uma proposta de modelo de negócios, utilizando a ferramenta Canvas Business Model (OSTERWALDER; PIGNEUR, 2010). Este exercício, ainda que parcial, se mostrou relevante para apontar considerações de negócio que podem impactar diretamente a experiência do serviço pelos usuários.

\section{RESULTADOS}

Durante a fase de entendimento e pesquisa inicial do projeto, foram analisadas nove empresas prestadoras de serviços voltados para idosos e moradia. A análise permitiu identificar duas categorias principais de assistência: personalizada, ou seja, serviços que buscam conhecer e entender o idoso como indivíduo, direcionando o atendimento conforme cada caso. E a assistência padronizada, serviços que são voltados para idosos em geral. Podemos ainda utilizar uma segunda classificação de importância, para assistências com foco residencial ou foco em saúde. 
Nota-se que a maioria dos serviços de assistência em saúde são voltados para um perfil de idoso que requer cuidados especiais, conferindo um caráter de atendimento mais personalizado, devido a uma internação domiciliar, monitoramento de doenças, curativos, medicações e/ou cuidadores. Já para as assistências residências, o forte são as empresas de seguros, poucas possuem uma cobertura voltada especificamente para idosos e em geral são serviços padronizados e burocratizados.

Diante deste cenário de mercado, aliado ao estudo sobre perfil do idoso e envelhecimento, percebe-se uma carência de serviços personalizados voltado para idosos em boas condições de saúde e a oportunidade de integrar os modelos de assistências residenciais e de saúde, visando uma abordagem de cuidados e atenção ao idoso, com foco na segurança, na qualidade de vida e humanização do atendimento.

Em paralelo a coleta de informações, por meio de ferramentas empáticas, foi realizada uma pesquisa com potenciais usuários do novo serviço, caracterizados através de dois perfis de personas, ambas com foco em idosos saudáveis e ativos, com um certo nível de independência e autonomia, sendo o primeiro perfil focado na faixa etária dos 60-79 anos e o segundo perfil de idosos na faixa dos 80 anos em diante.

As interações com os usuários ocorreram por meio de visitas nas residências, com o objetivo de conhecer sua rotina e atividades, bem como configuração da residência e histórias acerca dos temas: Segurança e Acessibilidade, Bem-Estar e Saúde, Lazer e Social.

Por meio dessa observação, constatou-se que os idosos mais novos, ou seja, a partir dos 60 anos, estão mais conscientes sobre aspectos preventivos tanto de saúde quanto de segurança, por estarem entrando em uma nova etapa da vida na qual entendem que precisam revisar diversos hábitos, mas nem sempre sabem o que e como fazer. Em geral possuem maior autonomia nas decisões, não sendo tão dependentes dos familiares. Ao contrário do perfil de idosos acima de 80 anos que já contam mais com familiares para acompanhamento médico, entre outros cuidados, além de apresentarem comportamentos de territorialidade e serem mais resistentes a mudanças.

Quando se trata de acidentes em casa, os idosos mais velhos relataram ter medo de acidentes domésticos como quedas e queimaduras, apontando também dificuldades principalmente no banheiro e na cozinha, como sendo os cômodos de maior risco, uma vez que para alcançar utensílios ou se equilibrar, eles utilizam de improvisações como subir em uma cadeira e neste momento que os acidentes ocorrem. Nota-se nesse perfil acima dos 80 anos ainda uma cultura de não querer incomodar e até mesmo uma certa teimosia. Ao contrário dos novos idosos que estão mais dispostos a adequar seu estilo de vida, incluindo sua moradia, ao seu melhor uso.

Também foi observado que os idosos em geral buscam se manter ativos de diversas formas e dentro de casa destacam-se atividades do carácter hobbies como jardinagem, leitura e costura. A principal diferença se dá no aspecto social, onde nota-se que os idosos acima de 80 anos em geral vivem em maior isolamento, muitas vezes sozinhos e com pouco auxílio nas atividades do dia a dia como limpeza, preparo de refeições, fazer compras, entre outros. De qualquer forma, assim como observado em campo e na "desk research" os idosos são mais carentes de convívio social e atividades de lazer em geral, tornando os momentos em que recebem atenção altamente valorizados. 
A partir da compreensão das demandas dos usuários e do cenário de mercado, foi possível identificar oportunidades de inovação em serviços voltados para moradia de idosos. A principal diz respeito à sensibilização e preparação de idosos e seus familiares sobre a necessidade de prevenção de acidentes como quedas e lesões.

Deste modo, foram estabelecidos requisitos para a configuração do novo serviço, apontando como Unidade de Satisfação a segurança e qualidade de vida do idoso em sua moradia:

- Prevenir acidentes com os idosos dentro de casa e promover seu bem-estar;

- Conscientizar idosos e familiares sobre importância da moradia segura;

- Avaliar a moradia do idoso identificando fatores de risco, nível de segurança e qualidade;

- Propor soluções para intervenção e correção dos pontos identificados na avaliação e seu devido impacto na melhoria e classificação de segurança;

- Estabelecer critérios de avaliação e classificação para a moradia;

- Personalização de quais coberturas e assistências o idoso irá optar, por motivos financeiros e grau de mudança e/ou impacto na moradia;

- Estabelecer um atendimento acolhedor e humanizado, para fortalecer confiança e construir relacionamento;

O novo conceito é voltado aos idosos com mais de 60 anos e apresenta potencial de contribuir para a melhora da qualidade de vida, independência funcional e conforto do idoso dentro da sua própria residência.

Figura 2. Etapas da experiência do serviço. Fonte: Baseado na pesquisa realizada

$\begin{array}{ll}\text { Apresentação } & \text { Ao entrar para um plano de saúde ao idoso, o usuário é convidado a realizar } \\ \text { um check-up funcional. } & \text { Espaço Elo: ambiente modelo, com decoração acolhedora e educativa, com } \\ & \text { exemplos de ambientes residenciais adaptados para maior segurança do } \\ & \text { idoso. Utilizado para recepção e atendimento do idoso. } \\ & \text { Gestor de Cuidados: especialista em gerontologia e que será o responsável } \\ \text { pelo acompanhamento do usuário ao longo de todo o serviço. } & \\ & \text { Responsável por introduzir junto ao usuário a necessidade de cuidados com } \\ & \text { segurança da moradia através de uma visão integrada de saúde e qualidade de } \\ & \text { vida. } \\ & \text { Médico Gestor: principal médico do usuário, sendo responsável por seu } \\ & \text { acompanhamento de saúde e endosso para realização do check-up da moradia } \\ \text { como parte do serviço de check-up. }\end{array}$

Avaliação Consiste no check-up da moradia.

Gestor de Cuidados: especialista visita a casa do usuário para uma avaliação dos cômodos, identificando pontos críticos e oportunidades de melhorias, através de conversas e observação.

Diagnóstico

Resultados e implementação das adaptações e melhorias.

Gestor de Cuidados: apresenta os resultados da avaliação e recomenda a implantação do Kit Básico de Cuidados.

Plano de Cuidados: consiste no relatório de avaliação da residência, apontando pontos críticos, recomendações de cuidados e é aprovado pelo Médico Gestor.

Kit Básico Segurança: compreende barras de apoio, antiderrapante de tapetes e luz de emergência. Estes itens são voltados a prevenção dos principais tipos de acidentes domésticos. 
Com base nos estudos realizados, identificamos a importância de integrar o novo serviço para a moradia à um serviço de saúde do idoso, na forma de um programa de assistência ou checkup anual, promovendo melhor qualidade de vida de seus segurados e consequentemente, reduzindo os riscos de acidentes e doenças. Trata-se de uma abordagem de cuidado orientada a prevenção, levando em consideração a capacidade funcional do indivíduo e a necessidade de adequação ambiental de sua residência.

Para tangibilizar o novo conceito, principalmente para as atividades de prototipação de validação com os usuários, foi criada uma identidade para o serviço, chamado "Elo Care Moradia Segura", traduzida visualmente ao longo dos principais pontos de contato mapeados, desde material de comunicação com o usuário até a ambientação do espaço de atendimento.

\section{CONSIDERAÇÕES FINAIS}

Muito embora continue sendo importante o desenvolvimento de artefatos físicos que contribuam para a melhoria da qualidade de vida do idoso no ambiente construído, o estudo apontou para a premente necessidade de direcionamento de esforços também para o Design de Serviço. O estudo de campo também apontou que a pauta de criação para os artefatos orientados à acessibilidade e segurança dos idosos pode ser advinda do Design de Serviço, o que amplia as chances de os mesmos terem maior efetividade tanto na relação com o usuário como na pertinência ao modelo de negócio preconizado. A partir da compreensão das demandas dos usuários foi também possível identificar oportunidades de inovação em serviços voltados para moradia de idosos. A principal diz respeito à sensibilização e preparação de idosos e seus familiares sobre a necessidade de prevenção de acidentes como quedas e lesões.

Para o conceito desenvolvido identificou-se a importância de integrar o novo serviço com outros serviços presentes no cotidiano do idoso, como os serviços de saúde. Trata-se de uma abordagem de cuidado orientada a prevenção, levando em consideração a capacidade funcional do indivíduo (perda de peso e massa muscular, déficit cognitivo, alterações de mobilidade, entre outros) e a necessidade de adequação ambiental de sua residência (facilitadores e obstáculos, características físicas e sensoriais, orientações e reparos). Desta forma, o serviço proposto tem como premissa a integração a plano de saúde do idoso, na forma de um programa de assistência ou check-up anual, promovendo melhor qualidade de vida de seus segurados e consequentemente, reduzindo os riscos de acidentes e doenças.

\section{REFERÊNCIAS}

AGNELLI, Luciana Bolzan. Avaliação da acessibilidade do idoso em sua residência / Luciana Bolzan Agnelli. -- São Carlos: UFSCar, 2012.

BITNER, Mary Jo; OSTROM, Amy L. e MORGAN, Felicia N. Service Blueprinting: a practical technique for service innovation. California Management Review. Vol. 50, no. 3, spring, 2008.

DAMÁZIO, Vera et FREIRE, Karine. Design de serviços: conceitos e reflexões sobre o futuro da disciplina. PUC RIO; UNISINOS (2010). 
EVENSON, SHELLEY. Theory and Method for Experience Centered Design. Jan 21, p.3. 2006.

MAGER, BIRGIT. Service Design as an emerging field. In: (Ed.). Designing Services with innovative methods. Keuruu: University of Art and Design Helsinki, v.1, 2009. Service Design as an emerging field, p.28-43,

MORAES, Edgar Nune. Avaliação multidimensional do idoso: instrumentos de rastreio/ Edgar Nunes Moraes. - Belo Horizonte: Follium, 2008 64p il.

MORITZ, STEFAN. Service Design: pratical acess to an evolving field. Köln International School of Design, University of Applied Sciences Cologne, 2005. 125 p.

OSTERWALDER, A.; PIGNEUR, Y. Business Model Generation (John Wiley \& sons, Eds.). p.278 p. New Jersey - USA, 2010.

ROHRBACH, B. (1953) Kreativ nach Regeln - Method 635, eine neue Technik zum Losen von Problemen.

SCHLEICHER, D. J., JONES, P. H., \& KACHUR, O. (2010) Bodystorming as embodied designing. Interactions, 17, 47-51.

SERVICE DESIGN TOOLS. Tool Storytelling. Disponível em: www.servicedesigntools.org/ tools/9 Último acesso em: 23 de julho de 2017.

TUKKER. Arnold. TNO Institute of Strategy, Technology and Policy, The Netherlandas. Eight types of Product Service System: Eight Ways To Sustainability? Experiences from Suspronet. Published online in Wiley Interscience ( www.interscience.wiley.com). DOI 10.1002/bse.414 (2004). 